\title{
Clinical Approach to Statin-Induced Adverse Muscle Events: Challenges and Solutions
}

\section{Kwame Nuako*}

University of Tennessee Health Science Center, USA

*Corresponding author: Kwame Nuako, University of Tennessee Health Science Center, USA, Tel: 731-819-0286; E-mail: knuako@uthsc.edu

Received date: December 21, 2017; Accepted date: February 28, 2018; Publication date: March 8, 2018

Citation: Nuako K (2018) Clinical Approach to Statin-Induced Adverse Muscle Events: Challenges and Solutions. J Prev Med Vol.3 No.2:9.

Copyright: (C) 2018 Nuako K. This is an open-access article distributed under the terms of the Creative Commons Attribution License, which permits unrestricted use, distribution and reproduction in any medium, provided the original author and source are credited.

\section{Abstract}

Statin-associated adverse muscle events play an important role in statin non adherence and discontinuation. Aspects of this clinical challenge including a lack of a uniform definition of statin-induced myalgia, the "nocebo" effect and its' role in statin-induced adverse muscle events, pre-existing medical and lifestyle factors that can affect statin therapy, and restarting statin use in patients after prior discontinuation of statin therapy due to adverse muscle effects.

This paper applies the recommendations of the National Lipid Association's Statin Muscle Safety Task Force along with other studies to propose solutions for these challenges that statin-induced adverse muscle events present. Future large randomized controlled trials will be necessary for validation of these clinical interventions.

Keywords: Statins; Statin-induced myalgia; Nocebo effect; Statin rechallenge

\section{Abbreviations:}

AMEs: Adverse muscle events

\section{Introduction}

HMG-CoA inhibitors (statins) have been demonstrated to effectively reduce the morbidity and mortality rates of cardiovascular disease, the leading cause of death in the United States [1]. Despite these benefits, adherence to statin therapy remains suboptimal (under $50 \%$ continuation within 1 year) with large scale studies suggesting that adverse muscle effects (AMEs) are the primary reason for discontinuation of statins amongst former statin users [2].

There are many challenges that clinicians face when approaching statin-induced AMEs. This includes [1] lack of a uniform definition of statin-induced myalgia [2] the role of "nocebo effect" in statin use [3] pharmacological and lifestyle factors that can affect statin therapy and [4] whether to restart statin after initial discontinuation due to presumed AMEs. This paper uses recommendations from the National Lipid Association's Statin Muscle Task Force and research from randomized controlled trials to address and propose solutions to each of these challenges.

\section{Development of uniform definition of myalgia}

Statins are generally well-tolerated with a 2014 meta-analysis of 26 randomized trials that included 113,695 participants found that muscle aches or pains were reported by $12.7 \%$ of those assigned to statins and $12.4 \%$ of placebo subjects, suggesting a difference of only $0.3 \%$ [4].

These findings suggest that adverse muscle symptoms that may have been attributed to statins could in fact have other etiologies. One major confounder in the assessment of statinassociated adverse muscle events is that there is an absence of a uniform and validated definition of myalgia [5].

Currently, most clinical trials that examine statin associated muscle pain use a self-reported system. In addition, the use of CK as a measurement of statin-induced myalgia is complicated by genetic factors as well as the patients' baseline activity levels [6].

The National Lipid Association's Statin Muscle Task Force has a suggested scoring system based on three specific criteria [7]. The clinical symptoms index assigns points based on specific clinical manifestations derived from studies that indicate that muscle symptoms attributable to statin therapy typically manifest as symmetric large muscle myopathies, [2] the temporal pattern index is derived from studies that suggest that symptoms of myalgia are more likely to occur within the first month of treatment, and thus assigns points based on the time frame in which symptoms begin [3] dechallenge and challenge index is based on studies that suggest that taking subjects off and back on medication can help determine relation of the pain to the statin.

Such a study could help further elucidate the difference between statin-induced myalgias and muscle pain of other etiologies and thus allow physicians to educate patients upfront 
about the probable sources of pain if this were to arise later in treatment [6].

Table 1: Proposed myalgia index by national lipid association's statin muscle task force [7].

\begin{tabular}{|c|c|}
\hline \multicolumn{2}{|l|}{ Proposed statin myalgia clinical index score } \\
\hline \multicolumn{2}{|c|}{ Clinical symptoms (new or increased unexplained muscle symptoms) } \\
\hline \multicolumn{2}{|l|}{ Regional distribution/pattern } \\
\hline Symmetric hip flexors/thigh aches & 3 \\
\hline Symmetric calf aches & 2 \\
\hline Symmetric upper proximal aches & 2 \\
\hline Non-specific asymmetric, intermittent & 1 \\
\hline \multicolumn{2}{|l|}{ Temporal pattern } \\
\hline Symptoms onset $<4$ weeks & 3 \\
\hline Symptoms onset $<4$ weeks & 3 \\
\hline Symptoms onset $4-12$ weeks & 2 \\
\hline Symptoms onset $>12$ weeks & 1 \\
\hline \multicolumn{2}{|l|}{ Dechallenge } \\
\hline Improves upon withdrawal (<2 weeks) & 2 \\
\hline Improves upon withdrawal (2-4 weeks) & 1 \\
\hline Does not improve upon withdrawal ( $>4$ weeks) & 0 \\
\hline \multicolumn{2}{|l|}{ Challenge } \\
\hline Same symptoms reoccur upon rechallenge $<4$ weeks & 3 \\
\hline Same symptoms recoccur upon rechallenge $4-12$ weeks & 1 \\
\hline \multicolumn{2}{|l|}{ Statin myalgia clinical index score } \\
\hline Probable & 09-Nov \\
\hline Possible & 07-Aug \\
\hline Unlikely & $<7$ \\
\hline
\end{tabular}

This myalgia clinical index score requires validation in a prospective trial in which patients with previous myalgia would be randomized to placebo or statin and then crossed over to either statin or placebo to determine the reproducibility of symptoms [6] is described in Table 1.

\section{Nocebo effect}

As previously mentioned, the difference in rates of myalgia between patients receiving placebo and those receiving statin therapy in controlled trials is minimal [4]. Despite this there is still a "nocebo" effect that may be in place with statin therapy. This effect is described as an adverse event, usually purely subjective, that results from expectations of harm from a drug, placebo, other therapeutic intervention or a nonmedical situation [7]. These perceptions about adverse effects are reinforced by information on the Internet and in social media, as well as "health scares" propagated by broadcast and print media [7].
For a clinician, it is important to articulate to a patient who is starting on a statin that muscle aches are common symptoms in middle-aged and older people and that statin may not be the root cause of this pain. It should be made clear that severe statin induced rhabdomyolysis is rare, occurring in less than 1 in 1000 patients, and that this is easily detected by measuring CK levels. Clinicians can also emphasize the proven substantial benefits of statins in reducing morbidity and mortality, and that the prevalence of statins as a common cause of adverse muscle events may be exaggerated by internet forums and other media outlets [7].

Overall, effective communication by the clinician can help to counter pre-existing negative expectations of statins and can aid with adherence to statin therapy.

\section{Factors during statin therapy}

Though the muscle related risks of statin use are difficult to quantify, there are some risk factors that the clinician can monitor to minimize risk. 
The PRIMO (Prediction of Muscular Risk in Observational conditions) study was an observational study of muscular symptoms in an unselected population of 7924 patients receiving high dosage statin therapy. In the study, there were several independent risk factors for development of statininduced myopathy including age ( $>80$ years), alcoholism, hypothyroidism, consumption of large quantities of grapefruit juice ( $>1$ quart/day), or major surgery (in the perioperative period) [8]. Pharmacologically, the interaction of statins with other categories of medications can enhance the risk of myopathic events [9].

Exercise levels also have an impact on statin related myalgia rates [10]. In the PRIMO study, the incidence of muscle pain with statin therapy increased with the level of physical activity from $10.8 \%$ in those engaging in leisure-type physical activity to $14.7 \%$ in those regularly engaging in vigorous activity, suggesting that statin-associated muscle side effects are provoked by physical activity [8]. Some studies suggest that clinicians should discontinue statin doses should be modulated in in active patients, especially before a high intensity event like a marathon [10]. Development of specific exercise regimens for patients on statins may be indicated in the future.

\section{Statin rechallenge}

Even in a patient who has been intolerant of statin treatment, rechallenge is often successful. Most patients who discontinue one statin because of myalgia can tolerate a different statin [11]. A retrospective analysis done in affiliation with Brigham and Women's Hospital and Massachusetts General Hospital involved 2721 patients who had discontinued statins. These patients were "rechallenged" with a statin and 92.2 percent were able to remain on treatment 12 months after the initial discontinuation[11]. The Cleveland Clinic Preventive Cardiology Program found that of patients referred who were intolerant to two statins largely because of myalgia, $72.5 \%$ could successfully tolerate a third statin[12].

When clinicians look to rechallenge a patient who was previously intolerant to statins, there are several therapeutic options including adjustment of statin therapy by changing the type of statin administered or decreasing the dose of statin [11]. Several studies have evaluated non-approved statin dosing regimens in patients with statin induced muscle events. This approach has been based on the concept that some statins, such as rosuvastatin and atorvastatin, have a longer half-life (18-20 h). These statins may maintain their lipid lowering effect over a longer period of time, and thus be administered at wider time intervals [12].

\section{Non-statin medications}

When no statin is tolerated or the maximal tolerable dose of statins fails to reduce LDL cholesterol to target levels, non-statin therapies should be considered. Most of these second line therapies are less effective at reducing LDL cholesterol levels, have unfavorable side effects, or are expensive. The most common alternatives to statin therapy are ezetimibe, bile acid sequestrants, niacin, and the PSK9 inhibitors. In extreme cases, when both cardiovascular risk and LDL cholesterol are high despite maximal tolerable drug therapy, LDL apheresis can be used.

\section{Future Challenges}

Large randomized controlled trials will be necessary for validation of clinical interventions that could help reduce statin non-adherence secondary to adverse muscle events, especially the validated muscle adverse events clinical index. There are a multitude of factors that may precipitate statin induced adverse muscle events, and clinicians may not be able to gather all relevant information during the patient interview. Future genetic studies may help to implicate specific or sensitive markers for that are predictive of statin induced myalgia.

\section{Conclusion}

Hyperlipidemic patients who experience adverse events during treatment are more likely to discontinue statin therapy. Since statins are the only agents with a robust body of evidence proving reduction in clinical end points, every effort should be made to continue high-risk patients on a statin-based regimen. The recommendations in this paper based on prior research may help clinicians more effectively manage statin-induced adverse muscle events and maintain their patients on life-saving therapy.

\section{In summary:}

There is no uniform definition for statin-induced myalgia, thus no definitive clinical endpoint at which to discontinue or alter statin therapy. The National Statin Association created a potential clinical index for the objective rating of these side effects, but further studies are needed for validation of this index.

The nocebo effect may influence patient's viewpoints of statins before beginning therapy, leading to a higher rate of reported adverse muscle events. Articulation of the benefits and safety of statins can help to counteract this effect in patients that receive statin therapy

There are a multitude of factors that can precipitate adverse muscle events during statin therapy. Monitoring these conditions can lead to a lower discontinuation rate because of adverse muscle events for patients on statin therapy

Patients are generally able to tolerate a second statin after discontinuation of a statin due to AMEs. Clinicians can alter dosage amount and intervals in order to ensure a more successful statin rechallenge.

Even with these recommendations, statin AMEs still pose a significant clinical challenge. Future randomized trials will be needed to test the validity of some of the aforementioned recommendations, and future advancements in testing may identify markers that are specific or sensitive for detection of statin induced myalgia. 


\section{References}

1. LIPID Study Group (2002) Long-term effectiveness and safety of pravastatin in 9014 patients with coronary heart disease and average cholesterol concentrations: The lipid trial follow-up. Lancet 359: 1379-1387.

2. Cohen JD, Brinton EA, Ito MK, Jacobson TA (2012) Understanding statin use in America and gaps in patient education (USAGE): An internet-based survey of 10,138 current and former statin users. J Clin Lipidol 6: 208-215.

3. Phillips PS, Haas RH, Bannykh S, Hathaway S, Gray NL, et al. (2002) Statin-associated myopathy with normal creatine kinase levels. Ann Intern Med 137: 581-585.

4. Ganga HV, Slim HB, Thompson PD (2014) A systematic review of statin-induced muscle problems in clinical trials. Am Heart J 168: 6-15.

5. Banach M, Rizzo M, Toth PP, Farnier M, Davidson MH, et al. (2015) Statin intolerance-an attempt at a unified definition, position paper from an international lipid expert panel: This paper is also published in parallel in archives of medical science expert opinion on drug safety 14: 935-955.

6. Rosenson RS, Baker SK, Jacobson TA, Kopecky SL, Parker BA, et al. (2014) An assessment by the statin muscle safety task force: 2014 update. J Clin Lipidol 8: 58-71.
7. Tobert JA, Newman CB (2016) The nocebo effect in the context of statin intolerance. J Clin Lipidol 10: 739-747.

8. Bruckert E, Hayem G, Dejager S, Yau C, Bégaud B, et al. (2005) Mild to moderate muscular symptoms with high-dosage statin therapy in hyperlipidemic patients-the PRIMO study. Cardiovasc Drugs Ther 9: 403-414.

9. Chatzizisis YS, Koskinas KC, Misirli G, Vaklavas C, Hatzitolios A, et al. (2010) Risk factors and drug interactions predisposing to statininduced myopathy. Drug Saf 33:171-87.

10. Deichmann RE, Lavie CJ, Asher T, DiNicolantonio JJ, O'Keefe JH, et al. (2015) The interaction between statins and exercise: Mechanisms and strategies to counter the musculoskeletal side effects of this combination therapy. Ochsner J 15: 429-37.

11. Zhang H, Plutzky J, Skentzos S (2013) Discontinuation of statin in routine care settings: A cohort study. J Vasc Surg 58: 843.

12. Backes JM, Venero CV, Gibson CA, Ruisinger JF, Howard PA, et al. (2008) Effectiveness and tolerability of every-other-day rosuvastatin dosing in patients with prior statin intolerance. Ann Pharmacother 42: 341-346. 